Research Article

\title{
A Novel Spine Fixation System Made Entirely of Carbon-Fiber-Reinforced PEEK Composite: An In Vitro Mechanical Evaluation
}

\author{
Ofir Uri (iD, Yoram Folman, Gil Laufer, and Eyal Behrbalk \\ Department of Orthopedic Surgery, Hillel Yaffe Medical Center, Hadera, Israel \\ Correspondence should be addressed to Ofir Uri; ofiruri@gmail.com
}

Received 26 September 2019; Revised 31 March 2020; Accepted 27 April 2020; Published 9 June 2020

Academic Editor: Andreas K. Demetriades

Copyright ( 2020 Ofir Uri et al. This is an open access article distributed under the Creative Commons Attribution License, which permits unrestricted use, distribution, and reproduction in any medium, provided the original work is properly cited.

\begin{abstract}
Background. Semirigid spine fixation systems utilizing nonmetallic materials have emerged as a promising innovation to overcome the inherent disadvantages of metal instrumentation in spine surgery. This study tests the mechanical properties of a novel spine fixation system made entirely of carbon-fiber-reinforced PEEK (CFR-PEEK) composite material (CarboClear System, CarboFix Orthopedics Ltd., Israel). Methods. An in vitro mechanical evaluation of the CFR-PEEK CarboClear system was conducted in compliance with the American Society for Testing and Materials (ASTM) F1717, F2193, and F543 standards. Results. The mean bending yield load, bending ultimate load, and bending stiffness of the construct were $322 \mathrm{~N}, 363 \mathrm{~N}$, and $45 \mathrm{~N} / \mathrm{mm}$, respectively. All tested samples completed $5 \times 10^{6}$ dynamic cycles successfully, with no evidence of fatigue failure at increasing load levels, up to $83 \%$ of ultimate bending load. The mean torsional stiffness was $1.0 \mathrm{Nm} / \mathrm{deg}$ and the mean screw axial pull-out strength was 2,037 N. Conclusion. The CarboClear Pedicle Screw System has mechanical properties comparable to those of other commonly used titanium-made systems, with superior fatigue properties. The fatigue resistance, modulus of elasticity which is very similar to that of bone, radiolucency, and CT/MRI artifact-free feature of this spine fixation system made entirely of CFRPEEK may offer advantages over traditional spine fixation systems made of metal alloys.
\end{abstract}

\section{Introduction}

The use of rigid metal instrumentation in spine surgery has become common practice over recent decades [1]. Although such instrumentation has dramatically increased the union rate in spinal fusion $[2,3]$, evidence suggests that the stiffness of the metallic implants used far exceeds the requirements for successful fusion and may lead to postoperative complications such as adjacent level degeneration $[4,5]$, vertebral body osteopenia related to the load bearing effect of rigid instrumentation [6], and screw loosening, especially in osteoporotic patients [7]. Moreover, metal-induced scattering associated with the use of metal alloys in spinal fusion reduces the reliability of imaging modalities such as CT, MRI in the postoperative follow-up, and treatment [8].

Semirigid fixation systems that utilize nonmetallic materials were developed to address these inherent disadvantages of metal instrumentation [9]. One of the material alternatives is the thermoplastic polyetheretherketone (PEEK) biocompatible polymer. Biomechanical studies comparing rods of posterior spinal fusion systems made of Ti-alloy versus PEEK demonstrated that the PEEK-rod system provides increased load sharing along the anterior column and lower stress at the bone-screw interface. This potentially reduces the risk of adjacent level degeneration, vertebral body bone loss, and screw loosening $[9,10]$. Being radiographically translucent, spinal instrumentation with rods made of PEEK interferes less with postoperative imaging, thus enabling easier radiographic follow-up at the rod area $[9,10]$.

A remaining disadvantage of the semirigid PEEK-rod systems is its inability to provide sufficient primary stability to allow spinal fusion due to micromovements attributed to the lower strength and modulus of elasticity of the PEEK 
compared to metals [8-10]. In order to provide PEEK with more strength and stiffness, a composite of PEEK-based matrix reinforced by carbon fibers (CFR-PEEK) has been introduced in orthopedic and spine surgery over the past years. It has been shown that orthopedic implants made of CFR-PEEK composite, with carbon fibers fraction of approximately $60 \%$ by volume, have mechanical properties (e.g., modulus of elasticity and fatigue strain) equivalent to that of cortical bone with promising results in spine surgery [11-14].

The CarboClear Pedicle Screw System (CarboFix Orthopedics Ltd., Herzliya, Israel) is a novel spine fixation system composed entirely of CFR-PEEK composite (i.e., rods and pedicle screws).

Preliminary reports of the CarboClear system for the treatment of spine tumors were recently published and showed outcomes comparable to the standard titanium system in terms of complications, stability, and functional scores $[13,14]$.

This study investigates the mechanical properties of the CarboClear system. To our knowledge, no previous study has evaluated the mechanical properties of the spine fixation system made entirely of CFR-PEEK composite. (Figure 1).

\section{Materials and Methods}

The study was conducted at the manufacturer's laboratories, CarboFix Orthopedics Ltd. (Herzliya, Israel), who provided financial support for the study, but was not involved in the manuscript writing and editing. All experiments were conducted under the strict protocols of the American Society for Testing and Materials (ASTM).

The CFR-PEEK specimens tested comprised $6.5 \mathrm{~mm}$ polyaxial pedicle screws with $6.0 \mathrm{~mm}$ straight rods and their locking elements. The manufacturer instructions were strictly kept throughout implant assembly.

The following mechanical tests were performed:

(1) Static axial compression bending test: the test was performed according to the ASTM F1717 standard [15], simulating a vertebrectomy model via a large gap between two ultrahigh-molecular weight polyethylene (UHMWPE) blocks simulating two vertebrae. Six CFR-PEEK constructs were tested, each comprising four pedicle screws and two rods. The apparatus included two UHMWPE blocks (with tensile breaking strength of $40 \pm 3 \mathrm{MPa}$ ), mounted by metal hinge pins to side supports on the test machine (Testometric M350-10 kN, Testometric Ltd., UK) and attached to the actuator and to the load cell. The upper side support-upper UHMWPE block construct and the lower side support-UHMWPE block construct were aligned. The center axis of each hinge pin was perpendicular to and aligned with the load axis of the test machine. As per ASTM F1717 recommendation, the active length of the longitudinal element (rod) was set at $76 \mathrm{~mm}$ and the block moment arm at $40 \mathrm{~mm}$ (Figure 2). Loading rate was $5 \mathrm{~mm} / \mathrm{min}$. Test plots of load versus displacement were generated for each assembly tested and the mode of failure of each construct was documented.

(2) Fatigue axial compression bending test: the test was performed according to the ASTM F1717 protocol. Six CFR-PEEK constructs were tested, each comprising four pedicle screws and two rods. A similar apparatus as for the static axial compression bending test was utilized. The samples were tested using an Instron 8871 fatigue system. The samples were evaluated at a number of increased load levels (defined as a percentage of the average ultimate axial compression bending load found at the static axial compression bending test described above, i.e., 50\%, $75 \%)$. The constructs were tested in phosphatebuffered saline solution at a temperature of $37^{\circ} \mathrm{C}$. The cycle rate was set at $2 \mathrm{~Hz}$. The endpoint of the test was defined as construct failure or completion of $5 \times 10^{6}$ cycles without failure. Plots of load vs. number of cycles and displacement vs. number of cycles were generated (Figure 3).

(3) Static torsion test: again, the test was performed according to the ASTM F1717 protocol, using a similar apparatus with the addition of aluminum blocks between the UHMWPE blocks and the base plate to stop rotation around the hinge pin. Four CFR-PEEK constructs were tested, each comprising four pedicle screws and two rods. The samples were tested using a tension/compression testing machine (Testometric M350-10 kN) at a rate of $6 \mathrm{deg} / \mathrm{min}$. Plots of torque vs. angular displacement were generated for each assembly tested (Figure 4).

(4) Screw axial pull-out strength test: this test was designed to measure the axial pull-out strength of the CFR-PEEK pedicle screw and was performed according to ASTM F2193 and ASTM F543 standards $[15,16]$. Four CFR-PEEK polyaxial pedicle screws (6.5 $\mathrm{mm}$ diameter, $35 \mathrm{~mm}$ length) were inserted into a rigid polyurethane foam block (density of $0.32 \mathrm{~g} / \mathrm{cm} 3$ ), a widely used substitute to cadaveric bone with more uniform material properties [17]. The construct was mounted onto the Testometric M350-10 kN test machine. A constant tensile load was applied at a rate of $5 \mathrm{~mm} / \mathrm{min}$ along the axis of the screw until the screw was released from the test block or broke (Figure 5).

\section{Results}

(1) Static axial compression bending test: a total of six CFR-PEEK construct samples, with $6.5 \mathrm{~mm}$ pedicle screws and $6.0 \mathrm{~mm}$ rods, were tested according to the ASTM F1717 protocol. The test results are summarized in Table 1. The failure mode for all the CFRPEEK samples tested was rotational slip of the rodscrew link.

(2) Fatigue axial compression bending test: a total of six CFR-PEEK construct samples with $6.5 \mathrm{~mm}$ pedicle screws and $6.0 \mathrm{~mm}$ rods were tested according to the 


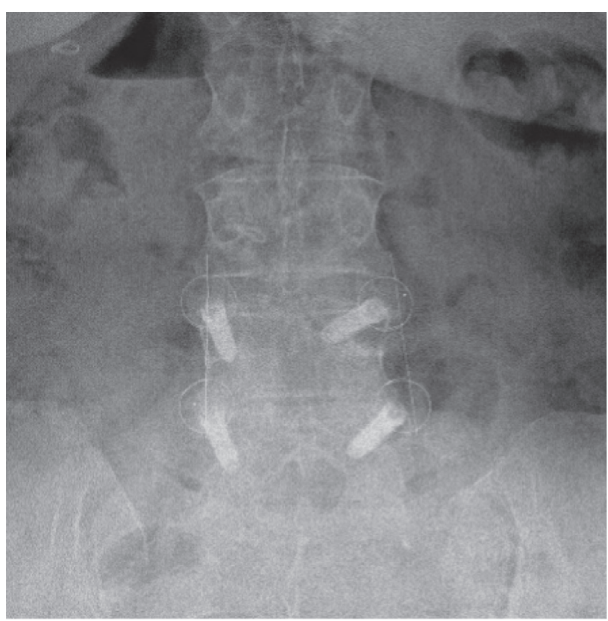

(a)

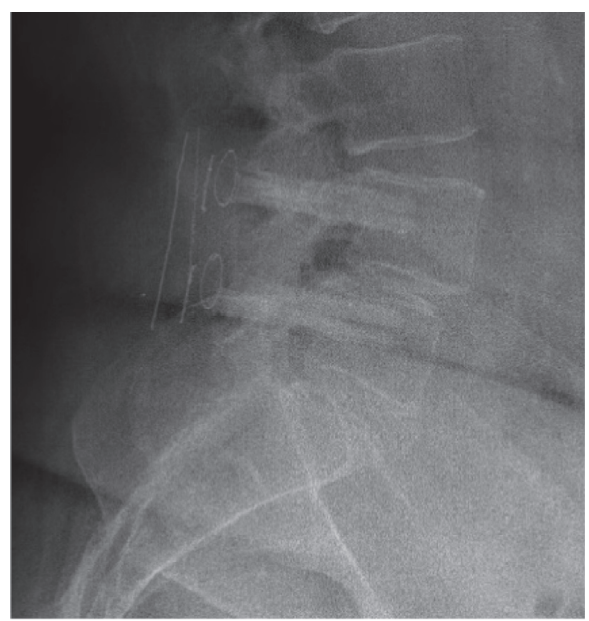

(b)

Figure 1: The CarboClear spine fixation system. (a) Anterior-posterior and (b) lateral lumbar spine radiographs of a 64-year-old female at 3 -year follow-up after spinal fusion with the radiolucent carbon-fiber-reinforced PEEK composite CarboClear system.

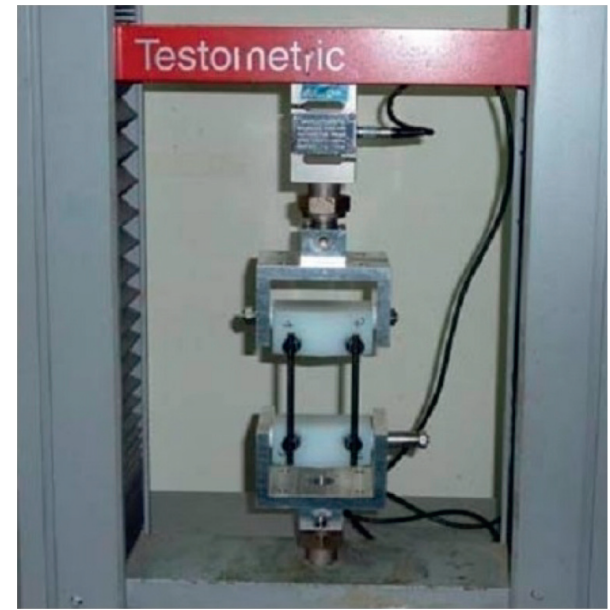

FIgURE 2: Static axial compression bending test performed in compliance with the ASTM F1717 standard, using a Testometric M350-10 kN testing machine, and simulating a vertebrectomy model via a large gap between two UHMWPE blocks simulating two vertebrae.

ASTM F1717 protocol. The test results are summarized in Table 2. No failure occurred at increasing load levels up to (and including) $300 \mathrm{~N}$ (83\% of ultimate axial compression bending load) and the tested samples completed $5 \times 10^{6}$ cycles successfully with no evidence of fractures, loosening of interconnections, plastic deformation of components or the construct, or other signs of failure. Microscopic examination of the components surface using a digital microscope, with a magnification range of $\times 20$ to x200 (Keyence VHX-700F series, Keyence Co., IL, USA), did not reveal damage, such as cracks, delamination, or scratches. Construct measurements taken at the completion of $5 \times 10^{6}$ cycles revealed no change in the effective length of the rods (the centerto-center distance between two longitudinally connected locking elements remained as $76 \mathrm{~mm}$ ), indicating that no permanent bending of the rods occurred. In all constructs, the four connections were intact, with no notable damage or loosening. The distance between the superior surface of the tulip and the rod was measured for all four interconnections in each construct and was found to be within the required range, indicating that no loosening/disassembly of the components occurred during the test. Also, the distances between the UHMWPE blocks at various points were measured for each construct, demonstrating that the blocks remained parallel to each other (i.e., indicating no slippage of the screw heads or bending of the rods has occurred). At a load level of $343 \mathrm{~N}$ ( $95 \%$ of ultimate axial compression bending load), the tested specimen failed after 150 cycles by slippage of the polyaxial screw head within the screw tulip.

(3) Static torsion test: a total of four CFR-PEEK construct samples, with $6.5 \mathrm{~mm}$ pedicle screws and $6.0 \mathrm{~mm}$ rods, were evaluated according to the ASTM F1717 standard. The results are presented in Table 3. The failure mode was slippage of the spherical screw head relative to the rod (slippage of the spherical screw head within the tulip).

(4) Screw axial pull-out strength test: a total of four samples of CFR-PEEK polyaxial pedicle screws (6.5 $\mathrm{mm}$ diameter, $35 \mathrm{~mm}$ length) were tested according to ASTM F2193 and ASTM F543 standards as applicable. Table 4 summarizes the test results. Failure in all the cases involved the polyurethane foam. No failure was detected in any of the screws.

\section{Discussion}

Spine fixation systems made of composite materials such as CFR-PEEK have emerged recently as promising innovations 


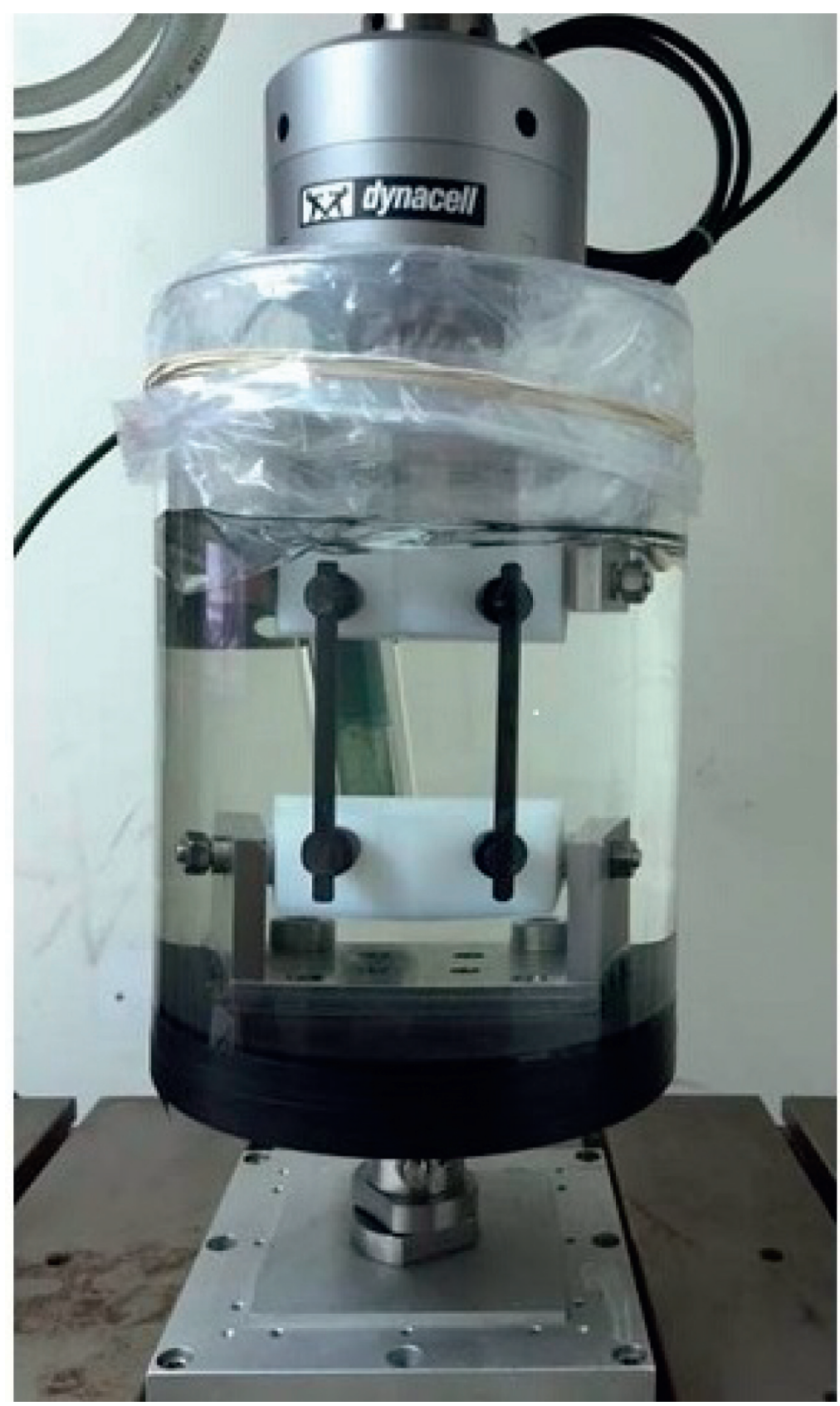

FIGURE 3: Fatigue axial compression bending test performed in compliance with the ASTM F1717 standard, using an Instron 8871 fatigue system testing machine, in phosphate-buffered saline solution setup at $37^{\circ} \mathrm{C}$.

in spine surgery. This study is the first to test the mechanical properties of the CarboClear system, a novel spine fixation system made entirely (screws and rods) of CRF-PEEK composite material.

We tested the CFR-PEEK pedicle screw system using various mechanical tests according to the ASTM standards in order to assess whether its mechanical properties are comparable with published data on other commonly used metal spine fixation systems.

The mean bending yield load and bending ultimate load of the CFR-PEEK system were $322 \mathrm{~N}$ and $363 \mathrm{~N}$, respectively, which are comparable to the values reported for the Moss Miami Ti system (DePuy, Warsaw, IN, USA) (299 N and $499 \mathrm{~N}$, respectively) and superior to those reported for the Synergy VLS Open system (Biomet Spine, Broomfield, CO, USA) (214 N and $292 \mathrm{~N}$, respectively) [18]. Bending stiffness of the CarboClear system was greater than the values reported for the titanium systems (CarboClear bending stiffness of $45 \mathrm{~N} / \mathrm{mm}$ compared to $24 \mathrm{~N} / \mathrm{m}$ and $33 \mathrm{~N} / \mathrm{mm}$ for the Moss Miami Ti and the Synergy VLS Open systems, respectively). This is an unexpected and less favorable finding, considering that one of the known advantages of CFR-PEEK implants is their semirigidity compared to metal implants that should enable more load sharing and avoid disadvantages of rigid instrumentation. A higher fraction of carbon fibers dispersed in the PEEK 


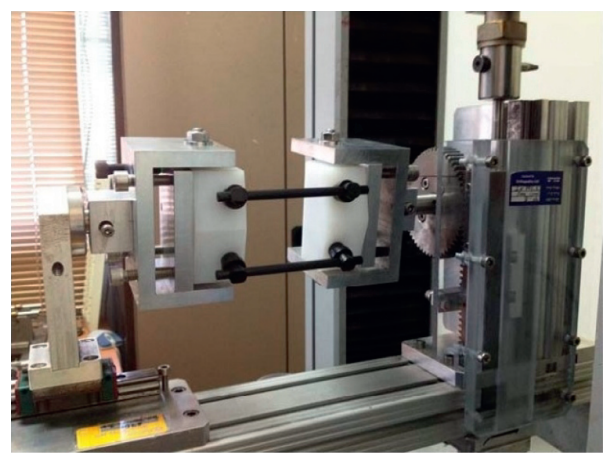

FIGURE 4: Static torsion test performed in compliance with the ASTM F1717 standard using a Testometric M350-10 kN testing machine at a rate of $6 \mathrm{deg} / \mathrm{min}$.

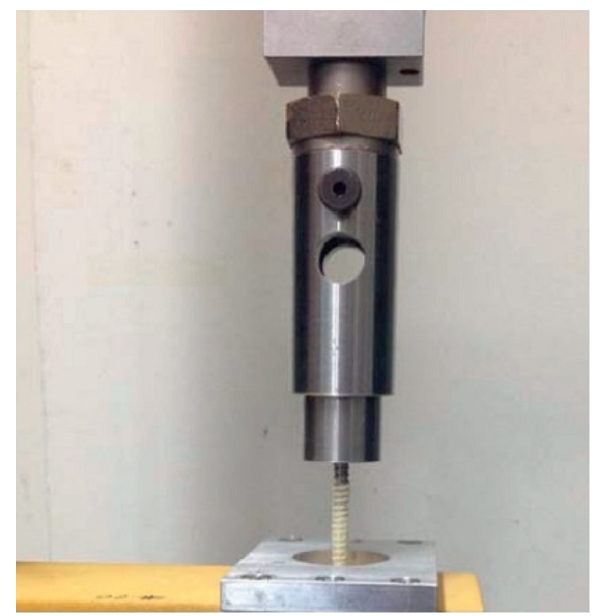

FIgURE 5: Screw axial pull-out strength test performed in compliance with ASTM F 2193 and ASTM F 543 standards using a Testometric M350-10 kN testing machine.

TABle 1: Static axial compression bending test results of the CarboClear system.

\begin{tabular}{lcccccc}
\hline Sample & $\begin{array}{c}\text { Bending yield } \\
\text { load (N) }\end{array}$ & $\begin{array}{c}\text { Bending } \\
\text { ultimate load } \\
(\mathrm{N})\end{array}$ & $\begin{array}{c}\text { Bending } \\
\text { stiffness } \\
(\mathrm{N} / \mathrm{mm})\end{array}$ & $\begin{array}{c}\text { Displacement at 2\% } \\
\text { offset yield (mm) }\end{array}$ & $\begin{array}{c}\text { Elastic } \\
\text { displacement } \\
(\mathrm{mm})\end{array}$ & $\begin{array}{c}\text { Bending } \\
\text { ultimate displacement (mm) }\end{array}$ \\
\hline 1 & 302 & 312 & 42 & 9.5 & 8.0 & 11.0 \\
2 & 310 & 325 & 50 & 7.7 & 6.5 & 8.3 \\
3 & 312 & 351 & 48 & 9.0 & 7.5 & 1.9 \\
4 & 350 & 401 & 44 & 9.3 & 7.8 & 1.8 \\
5 & 325 & 408 & 42 & 8.8 & 7.3 & 13.4 \\
6 & 335 & 381 & 46 & $8.8 \pm 0.8$ & $7.3 \pm 0.7$ & $11 \pm 1.8$ \\
Mean \pm SD & $322 \pm 18$ & $363 \pm 40$ & $45 \pm 3.2$ & & & \\
\hline
\end{tabular}

TABLE 2: Fatigue axial compression bending test results of the CarboClear system.

\begin{tabular}{|c|c|c|c|c|}
\hline Sample & $\begin{array}{l}\text { Load } \\
(\mathrm{N})\end{array}$ & $\begin{array}{l}\text { Percentage of ultimate bending load } \\
\qquad(\%)\end{array}$ & $\begin{array}{l}\text { No. of cycles to } \\
\text { failure }\end{array}$ & Mode of failure \\
\hline 1 & 200 & 55 & $5 \times 10^{6}$ & No failure, test terminated voluntarily \\
\hline 2 & 210 & 58 & $7.7 \times 10^{6}$ & No failure, test terminated voluntarily \\
\hline 3 & 230 & 63 & $5 \times 10^{6}$ & No failure, test terminated voluntarily \\
\hline 4 & 277 & 76 & $5 \times 10^{6}$ & No failure, test terminated voluntarily \\
\hline 5 & 300 & 83 & $6 \times 10^{6}$ & No failure, test terminated voluntarily \\
\hline 6 & 343 & 95 & 150 & $\begin{array}{l}\text { Slippage of the polyaxial screw head within the screw } \\
\text { tulip }\end{array}$ \\
\hline
\end{tabular}


TABle 3: Static torsion test results of the CarboClear system.

\begin{tabular}{lcccc}
\hline Sample & Yield torque $(\mathrm{Nm})$ & $\begin{array}{c}\text { Torsional stiffness } \\
(\mathrm{Nm} / \mathrm{deg})\end{array}$ & Angular displacement at 2\% offset yield (deg) Elastic angular displacement (deg) \\
\hline 1 & 12.4 & 0.9 & 16.1 & 14.2 \\
2 & 10.4 & 1.1 & 11.1 & 9.2 \\
3 & 12.4 & 1.2 & 12.4 & 10.2 \\
4 & 15.1 & 0.9 & 18.3 & 16.2 \\
Mean \pm SD & $12.6 \pm 1.9$ & $1.0 \pm 0.1$ & $14.4 \pm 3.3$ & $12.4 \pm 3.3$ \\
\hline
\end{tabular}

TABle 4: Screw axial pull-out test results of CarboClear pedicle screws ( $6.5 \mathrm{~mm}$ diameter, $35 \mathrm{~mm}$ length).

\begin{tabular}{lc}
\hline Sample & Axial pull-out strength $(\mathrm{N})$ \\
\hline 1 & 2151 \\
2 & 1901 \\
3 & 2041 \\
4 & 2057 \\
Mean \pm SD & $2037 \pm 103$ \\
\hline
\end{tabular}

matrix may explain this greater bending stiffness; however, this explanation is unlikely since the CarboClear system is designed to contain approximately $60 \%$ of carbon fibers by volume, which should yield stiffness similar to that of bone $[11,12]$ and not greater compared to metal implants. Further research may be required in order to clarify the nature of this finding.

The CFR-PEEK system showed no signs of fatigue at increasing cyclic axial compression loads up to $300 \mathrm{~N}(83 \%$ of its bending ultimate load) and successfully completed at least $5 \times 10^{6}$ cycles, with no evidence of mechanical failure. This meets the test acceptance criteria, which specifies that all samples tested at loads below $75 \%$ of the bending ultimate load should complete $5 \times 10^{6}$ cycles without failure. Both Moss Miami Ti and Synergy VLS Open systems showed inferior fatigue endurance and failed at $75 \%$ ultimate load after $0.04 \times 10^{6}$ and $0.98 \times 10^{6}$ cycles, respectively [18]. The superior fatigue endurance of the CFR-PEEK system may be attributed to the higher elasticity of the CFR-PEEK, as well as to the intrinsic properties of the composite material.

The torsional stiffness of the CFR-PEEK system $(1.0 \mathrm{Nm} /$ deg) was inferior to the values reported for several titanium systems, e.g., Globus Revolve polyaxial system (Globus Medical, Audubon, USA) (1.3 Nm/deg) [19], USS spine system (DePuy Synthes, Zuchwil, Switzerland) (2.2 Nm/deg) [19], and Moss Miami Ti system (1.8 Nm/deg) [20]. The failure mode of the CFR-PEEK specimens that we tested was a slippage of the spherical screw head relative to the rod (i.e., slippage of the spherical screw within screw tulip). A similar failure pattern, attributed to the coupling mechanism between the polyaxial screw head and its shaft, was found also in the Revolve and USS systems mentioned above $[19,20]$.

The axial pull-out strength of the CFR-PEEK $6.5 \mathrm{~mm}$ pedicle screws observed in our testing $(2,037 \mathrm{~N})$ is comparable to the pull-out strength of the Moss Miami $6.9 \mathrm{~mm}$ pedicle screw $(1,888 \mathrm{~N})$ and the Cotrel-Dubousset $6.5 \mathrm{~mm}$ pedicle screw (Medtronic Sofamor Danek, Memphis, TN) $(1,895 \mathrm{~N})$ [17]. All screw systems failed at the screw- polyurethane foam interface, with no structural damage to the screw itself.

Our adherence to the ASTM F1717 vertebrectomy model protocol may be considered as a limitation of the study, since the F1717 protocol has been shown to be a worst-case loading condition that may not actually represent loading within activities of daily living [21].

\section{Conclusion}

We demonstrated that the composite material spine fixation system, comprising CFR-PEEK pedicle screws and rods, has mechanical properties comparable to those of other commonly used titanium-made systems, with superior fatigue properties. The fatigue resistance, modulus of elasticity which is very similar to that of bone, radiolucency, and CT/ MRI artifact-free feature of spine fixation systems made entirely of CFR-PEEK may offer advantages over traditional spine fixation systems made of metal alloys. Continued clinical research with large patient cohort, variety of surgical indications, and long-term follow-up is required in order to evaluate the performance of the CarboClear system over time.

\section{Data Availability}

The data used to support the findings of this study are available from the corresponding author upon request.

\section{Disclosure}

The funders had no role in manuscript writing and editing.

\section{Conflicts of Interest}

The authors declare that there are no conflicts of interest regarding the publication of this paper.

\section{Acknowledgments}

The study was conducted at the manufacturer's laboratories, CarboFix Orthopedics Ltd. (Herzliya, Israel), who provided financial support for the study.

\section{References}

[1] H. Yoshihara, "Rods in spinal surgery: a review of the literature,” The Spine Journal, vol. 13, no. 10, pp. 1350-1358, 2013. 
[2] C. M. Bono and C. K. Lee, "Critical analysis of trends in fusion for degenerative disc disease over the past 20 years," Spine, vol. 29, pp. 455-463, 2004.

[3] N. Boos and J. Webb, "Pedicle screw fixation in spinal disorders: a European view," European Spine Journal, vol. 6, pp. 2-18, 1997.

[4] J. M. Highsmith, L. M. Tumialán, and G. E. Rodts Jr, "Flexible rods and the case for dynamic stabilization," Neurosurgical Focus, vol. 22, pp. 1-5, 2007.

[5] M. D. Rahm and B. B. Hall, "Adjacent-segment degeneration after lumbar fusion with instrumentation: a retrospective study," Journal of Spinal Disorders, vol. 9, pp. 392-400, 1996.

[6] T. G. Craven, W. L. Carson, M. A. Asher et al., "The effects of implant stiffness on the bypassed bone mineral density and facet fusion stiffness of the canine spine," Spine, vol. 19, pp. 1664-1673, 1994.

[7] F. Galbusera, D. Volkheimer, S. Reitmaier, N. Berger-Roscher, A. Kienle, and H. J. Wilke, "Pedicle screw loosening: a clinically relevant complication?" European Spine Journal, vol. 24, no. 5, pp. 1005-1016, 2015.

[8] R. K. Ponnappan, H. Serhan, B. Zarda, R. Patel, T. Albert, and A. R. Vaccaro, "Biomechanical evaluation and comparison of polyetheretherketone rod system to traditional titanium rod fixation," The Spine Journal, vol. 9, no. 3, pp. 263-267, 2009.

[9] M. F. Gornet, F. W. Chan, J. C. Coleman et al., "Biomechanical assessment of a PEEK rod system for semi-rigid fixation of lumbar fusion constructs," Journal of Biomechanical Engineering, vol. 133, no. 8, 2011.

[10] F. Galbusera, C. M. Bellini, F. Anasetti, C. Ciavarro, A. Lovi, and M. Brayda-Bruno, "Rigid and flexible spinal stabilization devices: a biomechanical comparison," Medical Engineering \& Physics, vol. 33, no. 4, pp. 490-496, 2011.

[11] C. S. Li, C. Vannabouathong, S. Sprague, and M. Bhandari, "The use of carbon-fiber-reinforced (cfr) PEEK material in orthopedic implants: a systematic review," Clinical Medicine Insights: Arthritis and Musculoskeletal Disorders, vol. 8, pp. 33-45, 2015.

[12] D. J. Hak, C. Mauffrey, D. Seligson, and B. Lindeque, "Use of carbon-fiber-reinforced composite implants in orthopedic surgery," Orthopedics, vol. 37, no. 12, pp. 825-830, 2014.

[13] G. Tedesco, A. Gasbarrini, S. Bandiera, R. Ghermandi, and S. Boriani, "Composite PEEK/Carbon fiber implants can increase the effectiveness of radiotherapy in the management of spine tumors," Journal of Spine Surgery, vol. 3, no. 3, pp. 323-329, 2017.

[14] S. Boriani, G. Tedesco, L. Ming et al., "Carbon-fiber-reinforced PEEK fixation system in the treatment of spine tumors: a preliminary report," European Spine Journal, vol. 27, no. 4, pp. 874-881, 2018.

[15] V. K. Goel, M. M. Panjabi, A. G. Patwardhan, A. P. Dooris, and H. Serhan, "American Society for Testing and Materials. Test protocols for evaluation of spinal implants," The Journal of Bone and Joint Surgery (American), vol. 88, no. 2, pp. 103-109, 2006.

[16] A. Koistinen, S. Santavirta, and R. Lappalainen, "Apparatus to test insertion and removal torque of bone screws," Proceedings of the Institution of Mechanical Engineers, Part H: Journal of Engineering in Medicine, vol. 217, no. 6, pp. 503-508, 2003.

[17] C. C. Hsu, C. K. Chao, J. L. Wang, S. M. Hou, Y. T. Tsai, and J. Lin, "Increase of pullout strength of spinal pedicle screws with conical core: biomechanical tests and finite element analyses," Journal of Orthopaedic Research, vol. 23, no. 4, pp. 788-794, 2005.
[18] R. E. Stanford, A. H. Loefler, P. M. Stanford, and W. R. Walsh, "Multiaxial pedicle screw designs: static and dynamic mechanical testing," Spine, vol. 29, no. 4, pp. 367-375, 2004.

[19] D. Kubosch, E. J. Kubosch, B. Gueorguiev et al., "Biomechanical investigation of a minimally invasive posterior spine stabilization system in comparison to the Universal Spinal System (USS)," BMC Musculoskelet Disord, vol. 17, p. 134, 2016.

[20] D. Kok, P. J. Firkins, F. H. Wapstra, and A. G. Veldhuizen, "A new lumbar posterior fixation system, the memory metal spinal system: an in-vitro mechanical evaluation," $B M C$ Musculoskelet Disord, vol. 14, p. 269, 2013.

[21] L. La Barbera, F. Galbusera, H. J. Wilke, and T. Villa, "Preclinical evaluation of posterior spine stabilization devices: can the current standards represent basic everyday life activities?" European Spine Journal, vol. 25, no. 9, pp. 2909-2918, 2016. 